\title{
Endothelium of the Fetal Capillary
}

National Cancer Institute

\section{Source}

National Cancer Institute. Endothelium of the Fetal Capillary. NCI Thesaurus. Code C34162.

A continuous endothelium that forms one of four layers of the placental barrier separating maternal and fetal blood. 